\title{
Sharpening Occam's Razor - A Diagnostic Dilemma
}

\author{
Payal Sen, $M D^{1}$ \\ Uddalak Majumdar, $M D^{2}$ \\ Patrick Rendon, $M D^{1}$ \\ Ali Imran Saeed, $M D^{1}$ \\ Akshay Sood, MD' \\ ${ }^{1}$ University of New Mexico \\ Albuquerque, NM US \\ ${ }^{2}$ Cleveland Clinic Foundation \\ Cleveland, OH USA
}

\begin{abstract}
Objective: Physicians often search for Occam's Razor, that is, to have a single diagnosis explain all clinical manifestations in an individual patient. Herein, we describe a case which was significant for a dual clinical diagnosis, thus proving that Occam's razor may not always hold true.

Case Summary: A 22-year-old Caucasian man presented with 4 days history of fever, and dry cough. Chest $\mathrm{x}$-ray revealed a right middle lobe pneumonia. Mycoplasma IgM antibody titer was significantly elevated $(>1: 320)$, using the rapid diagnosis enzymeimmunoassay (EIA) test, and clinical course was complicated by rhabdomyolysis. He was treated with oral azithromycin for 5 days. The patient however returned to the ER in 2 weeks with similar symptoms and repeat chest $\mathrm{x}$-ray revealed a persistent right middle lobe infiltrate. Endobronchial biopsy revealed necrotizing granulomatous inflammation which stained positive for Histoplasma capsulatum. Serum complement fixation antibody test for Histoplasma demonstrated an elevated titer of 1:64. The patient was diagnosed to have an 'atypical pneumonia due to sub-acute Histoplasma capsulatum and acute Mycoplasma Pneumoniae infections, complicated by rhabdomyolysis.' Discussion: This case is unusual because the patient had an acute communityacquired atypical pneumonia from Mycoplasma pneumoniae, complicated by rhabdomyolysis, and also had subacute Histoplasma pneumonia. Physicians often search for Occam's Razor. However, following Hickam's dictum, we made the unusual diagnosis of concomitant lung infection in an immunocompetent host with Mycoplasma pneumoniae and Histoplasma capsulatum.

Conclusion: This was an immunocompetent patient who ran a complex, protracted, and unusual course of community acquired pneumonia. Often, the pursuit of additional or alternative diagnoses may require repeated and multiple invasive diagnostic sampling. Occam's razor may not always hold true.
\end{abstract}

\section{Introduction}

Occam's razor proposes that the simplest explanation is usually the correct one. However, in the science of medicine, simple solutions may be elusive. Often there is an 
incredibly complex constellation of symptoms co-occurring with one another, thereby confounding the scientific community. We described the diagnostic conundrums in managing our patient who ran a complex protracted course of community acquired pneumonia.

\section{Case}

A 22-year-old Caucasian male college student with no significant past medical history, initially presented to the University hospital in New Mexico, United States, with 4 days' history of fever, dry cough, and dyspnea. He had recently returned from a family vacation in Illinois and had spent several weeks fishing on the Mississippi river. Review of systems was negative for chest pain, headache, fever, chills, or night sweats. He denied any sick contacts. He did not smoke and did not use recreational drugs. His grandfather, who had been a heavy cigar smoker, had died of lung cancer.

His vital signs were significant for a body temperature of $100.6^{\circ}$ Fahrenheit, respiratory rate of 32 breaths per minute, pulse rate of $94 \mathrm{bpm}$, blood pressure of 130/82 millimeters of mercury, and pulse oximetry of 90 percent on room air. Physical examination demonstrated that he was in mild respiratory distress. Chest auscultation revealed decreased breath sounds over the right mid to lower lung field. The rest of his physical examination was otherwise unremarkable.

His laboratory tests revealed a normal complete blood count with a hematocrit of $40.5 \%$, white blood cell count of 8,200 cells per microliter, and platelet count of 263,000 per microliter. His electrolyte levels showed a serum sodium of $136 \mathrm{mEq}$ per liter, potassium of $3.4 \mathrm{mEq}$ per liter, chloride of $100 \mathrm{mEq}$ per liter, bicarbonate of $21 \mathrm{mEq}$ per liter, blood urea nitrogen of $15 \mathrm{mg} / \mathrm{dL}$ and creatinine of $0.9 \mathrm{mg} / \mathrm{dL}$. His blood glucose was normal at $98 \mathrm{mg} / \mathrm{dL}$. His urine analysis revealed $3+$ blood without red blood cells. His liver function tests demonstrated an elevated aspartate aminotransferase at 244 units per liter, elevated alanine aminotransferase at 72 units per liter, with normal total bilirubin, albumin, and alkaline phosphatase levels. His serum creatinine kinase (CK) was highly elevated at 26,000 units per liter (normal reference range 39-308 units per liter). His arterial blood gas at rest on room air at an elevation of 5500 feet above sea level showed acute respiratory alkalosis with a normal alveolar arterial gradient with a $\mathrm{pH}$ of $7.57, \mathrm{PaCO} 2$ of $28 \mathrm{mmHg}, \mathrm{PaO} 2$ of $77 \mathrm{mmHg}$, and bicarbonate of $22 \mathrm{mEq}$ per liter. His mycoplasma IgM antibody titer was significantly elevated ( $>1: 320)$ using the rapid diagnosis enzyme-immunoassay (EIA) test. Anti-mycoplasma pneumoniae IgA was also elevated. The urinary legionella and pneumococcal antigen levels, sputum culture, blood cultures, and urine toxicology screen were negative. Chest radiograph revealed a right middle and lower lobe pneumonia (Figure 1). 


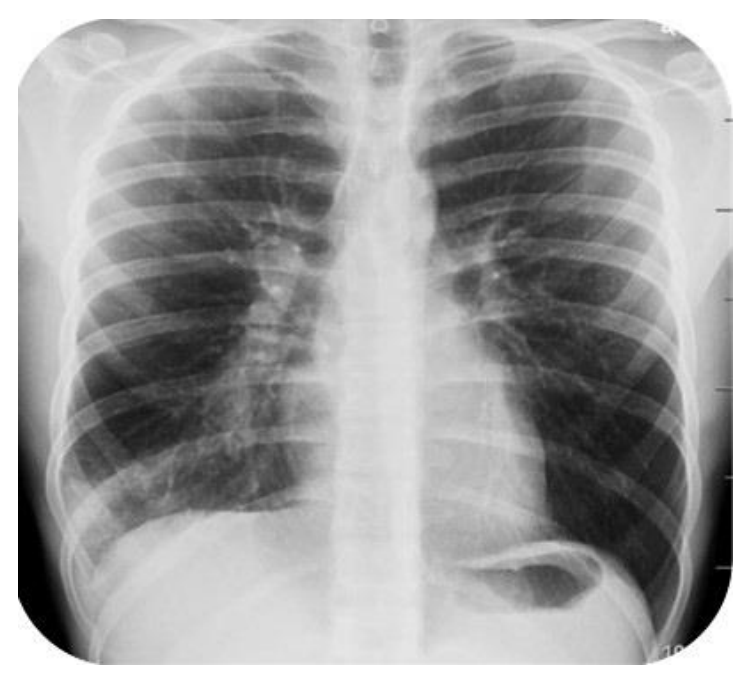

Figure 1. CXR revealed right mid and lower lobe pneumonia.

The patient was diagnosed with sepsis secondary to Mycoplasma pneumoniae infection of the lungs, with the added complication of rhabdomyolysis. He was treated with intravenous followed by oral azithromycin $500 \mathrm{mg}$ daily for 5 days and given intense hydration therapy. Within 48 hours, his low-grade fever subsided, CK decreased to 1000 units per liter, and the patient felt better. He was then discharged on Day 3 of hospitalization.

The patient however returned to the emergency department 2 weeks after discharge with persistent cough, chest discomfort, and loss of wellbeing. Repeat chest radiograph revealed a persistent right lower lobe infiltrate. Computed tomography (CT) scan of the chest revealed a right lower lobe consolidation with surrounding nodular opacities with a possible endobronchial lesion in the right lower lobe (Figure 2).
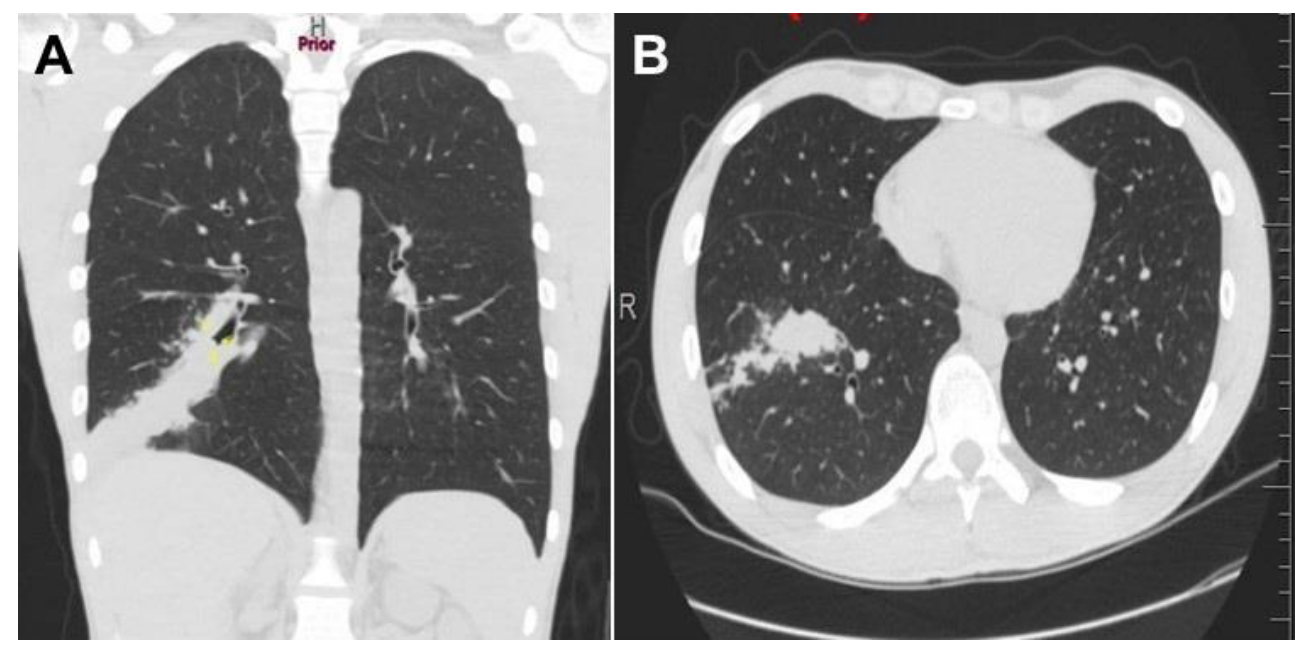

Figure 2. Panel A: Coronal view of thoracic CT scan showing right lateral basilar segment consolidation. Panel B: Axial view showing consolidation in the right lower lobe with surrounding nodular opacities. 
He underwent bronchoscopy which revealed a mass-like endobronchial lesion in the lateral basilar segmental bronchus of the right lower lobe (Figure 3).

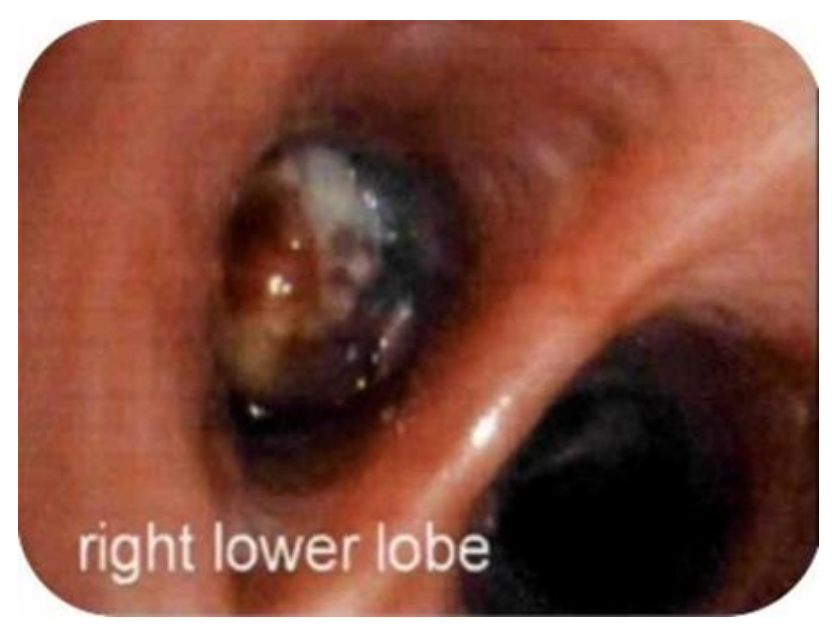

Figure 3. Bronchoscopy revealing a mass-like endobronchial lesion in a lateral segmental bronchus of the right lower lobe.

Endobronchial biopsy revealed necrotizing granulomatous inflammation and stained positive for the yeast form of Histoplasma capsulatum. Serum complement fixation antibody test for Histoplasma demonstrated an elevated titer of 1:64. Acid fast bacilli were not seen on smear or culture and cytology and histopathology tests did not reveal malignancy.

The patient was diagnosed with an atypical pneumonia due to sub-acute Histoplasma capsulatum and acute Mycoplasma Pneumoniae infections, complicated by rhabdomyolysis. The mycoplasma infection and rhabdomyolysis had already been treated and resolved. For the subacute pulmonary histoplasmosis, the patient was treated with 10 weeks of oral itraconazole. Post treatment clinic follow-up revealed resolution of symptoms and radiological abnormalities.

\section{Discussion}

Mycoplasma pneumoniae is a common causative pathogen for community-acquired pneumonia in both children and adults (1). Apart from respiratory tract symptoms, it is associated with a variety of extra-pulmonary manifestations (2). Recognizing this association can lead to timely diagnosis and treatment of both the mycoplasma infection and its complications. In this case report, we also want to highlight the fact that infection with endemic mycoses can often be mistaken for community acquired pneumonias, and thus having a high index of suspicion for fungal infection is very important, even in immunocompetent patients (3), to prevent a delay in treatment. Physicians often search for Occam's Razor, i.e., to have a single diagnosis explain all clinical manifestations in an individual patient. This case is significant because of a dual clinical diagnosis, thus proving that Occam's razor may not always hold true in an individual patient. 
Mycoplasma infection can cause several unusual extra-pulmonary manifestations such as hemolytic anemia, immune thrombocytopenic purpura, transverse myelitis, GuillainBarre syndrome, acute hepatitis and arthritis (4). Another lesser known complication of mycoplasma infection is rhabdomyolysis (5). Rhabdomyolysis is a syndrome caused by injury to the skeletal muscles, thereby resulting in leakage of myoglobin into blood (6). The classic triad of mycoplasma infection consists of myalgias, pigmenturia, and generalized muscle weakness, but this classic triad is seen in less than 10 percent of infected patients (7). Acute renal failure due to acute tubular necrosis as a result of mechanical obstruction by myoglobin is the most common complication, in particular if the serum CK level is $>16,000 \mathrm{IU} / \mathrm{l}$, which may be as high as 100,000 IU/I (8). In addition to mycoplasma infection, more common causes of rhabdomyolysis are trauma, immobilization, and recreational drug and alcohol use (9).

Other organisms known to cause rhabdomyolysis are Influenza $A$ and $B$ virus, Coxsackie virus, Epstein-Barr virus, Primary Human Immunodeficiency virus, Legionella species, Staphylococcus aureus, and Streptococcus pyogenes (9). With respect to Mycoplasma pneumoniae infection, a possible mechanism for rhabdomyolysis is the induction of inflammatory cytokines, such as tumor necrosis factor-alfa (TNF- $\alpha$ ) and interleukin-1 (IL-1), which may cause proteolysis of skeletal muscles (10).

The rapid and reliable diagnosis of Mycoplasma pneumoniae (Mp) enables the correct and prompt use of antibiotics. Methods for identifying $M p$ infection include culture, molecular detection of pathogen specific antigen or nucleic acid, and serological analysis (11). Each of these methods has its pros and cons. Culture is the definitive method for diagnosis and is critical for monitoring trends in epidemiology but is slow and requires specialized media and trained personnel (11). Although molecular methods for nucleic acid or antigen detection have emerged as the primary techniques for identification of MP pneumoniae in surveillance programs, adoption of these methods is still lagging behind in USA.

Serologic analysis can prove to be problematic due to poor sensitivity and specificity, and the inability to characterize the specific $M p$ strain. Having said that, most physicians in the United States continue to rely on serological testing in concordance with the IDSA guidelines (11). It is well known that a single serologic test is of limited value in the early diagnosis of mycoplasma pneumoniae since there are often no $\operatorname{lgM}$ antibodies in the early stage of infection, and these IgM antibodies may persist long after the infection (12). However, if these IgM antibodies are present along with anti-Mycoplasma pneumoniae $\lg \mathrm{A}$, it is usually indicative of recent primary mycoplasma pneumoniae infection (13). A single high Mp-specific antibody titer (> 1:320) has been regarded as a diagnostic marker of mycoplasma pneumoniae, although it is present in only about 30 percent of the patients (12). Since our hospital relies on serological testing, we tested for the specific Mycoplasma pneumoniae IgM and IgA, both of which were positive. The MP-specific antibody titer was also greater than 1:320, thus signifying it indeed was early MP infection. 
Symptoms of $M p$ infection generally resolve within 3-4 weeks after disease onset but can be shortened with antibiotic therapy; macrolides and doxycycline are the mainstay of this treatment (14). The mainstay for the prevention of pigment-induced acute kidney injury is the correction of volume depletion, prevention of intratubular cast formation, and the treatment of the underlying cause of rhabdomyolysis (4). This is done by aggressive fluid resuscitation resulting in increased renal blood flow and thus increasing the urinary flow with consequential wash out of partially obstructing tubular casts (4). Physicians will be served well to watch out for mycoplasma associated rhabdomyolysis in patients with atypical pneumonia and manifestations like myalgia, elevated aminotransferase levels, and myoglobinuria.

Moving on to the second teaching point, endemic mycoses like coccidioidomycosis, histoplasmosis, and blastomycosis are often overlooked causes for community acquired pneumonia, particularly when immunocompetent patients travel out of the endemic zones (15). Often, testing is not even performed until the patient has failed to improve on antibacterial therapy. Delays in recognition, diagnosis and proper treatment may lead to disastrous outcomes (3). Performance of fungal antigen testing on bronchial washings or lavage fluid may improve the sensitivity for diagnosis over microscopic examination and the speed of diagnosis over culture even though isolation of the fungus by culture remains the gold standard method for definitive diagnosis (16). In this case, our patient was previously treated as mycoplasma pneumonia, thus leading to prolonged symptom course from histoplasmosis.

This case is unusual because the patient had an acute community-acquired atypical pneumonia from Mycoplasma pneumoniae, complicated by rhabdomyolysis, and also had subacute Histoplasma pneumonia. Physicians often search for Occam's Razor, a principle from philosophy that when presented with competing hypothetical answers to a problem, one should select the one that makes the fewest assumptions. Countering Occam's Razor, Dr. John Hickam said "Patients can have as many diseases as they damn well please!" (17). Following Hickam's dictum, we made the unusual diagnosis of concomitant lung infection in an immunocompetent host with Mycoplasma pneumoniae and Histoplasma capsulatum.

\section{Conclusion}

With this case report, the authors wish to highlight two important teaching points. The first being that rhabdomyolysis is a serious but treatable extrapulmonary complication of Mycoplasma pneumoniae infection of the lungs. Having a high index of suspicion can limit treatment delay for rhabdomyolysis caused by mycoplasma infection and will therefore limit consequential morbidity like renal insufficiency. The second point that the authors wish to emphasize is that endemic fungal infection can often be mistaken for bacterial and viral community-acquired pneumonia in an immunocompetent host, particularly when they present with symptoms outside the endemic zone, thus delaying timely management. Hence one should have a high suspicion for fungal infection in immunocompetent hosts with unusual presentations such as history of travel to endemic 
zone, chronicity of symptoms, lack of response to therapy for community-acquired pneumonia, nodular lung lesions, and endobronchial abnormalities.

\section{References}

1. Hardy RD, Jafri HS, Olsen K, Hatfield J, Iglehart J, Rogers BB, Patel P, et al. Mycoplasma pneumoniae induces chronic respiratory infection, airway hyperreactivity, and pulmonary inflammation: a murine model of infection-associated chronic reactive airway disease. Infect Immun. 2002 Feb;70(2):649-54. [CrossRef] [PubMed]

2. Kawai Y, Miyashita N, Kato T, Okimoto N, Narita M. Extra-pulmonary manifestations associated with Mycoplasma pneumoniae pneumonia in adults. Eur $\mathrm{J}$ Intern Med. 2016 Apr;29:e9-e10. [CrossRef] [PubMed]

3. Hage CA, Knox KS, Wheat LJ. Endemic mycoses: overlooked causes of community acquired pneumonia. Respir Med. 2012 Jun;106(6):769-76. [CrossRef] [PubMed]

4. Gosselt A, Olijhoek J, Wierema T. Severe asymptomatic rhabdomyolysis complicating a mycoplasma pneumonia. BMJ Case Rep. 2017 Jul 26;2017. pii: bcr2016-217752. [CrossRef] [PubMed]

5. Khan FY, Sayed H. Rhabdomyolysis associated with Mycoplasma pneumoniae pneumonia. Hong Kong Med J. 2012 Jun;18(3):247-9. [PubMed]

6. Zimmerman JL, Shen MC. Rhabdomyolysis. Chest. 2013 Sep;144(3):1058-65. [CrossRef] [PubMed]

7. Zutt R, van der Kooi AJ, Linthorst GE, Wanders RJ, de Visser M. Rhabdomyolysis: review of the literature. Neuromuscul Disord. 2014 Aug;24(8):651-9. [CrossRef] [PubMed]

8. Allison SJ. Acute kidney injury: Macrophage extracellular traps in rhabdomyolysisinduced AKI. Nat Rev Nephrol. 2018 Mar;14(3):141. [CrossRef] [PubMed]

9. Bosch X, Poch E, Grau JM. Rhabdomyolysis and acute kidney injury. N Engl J Med. 2009 Jul 2;361(1):62-72. [CrossRef] [PubMed]

10. Giannoglou GD, Chatzizisis YS, Misirli G. The syndrome of rhabdomyolysis:

Pathophysiology and diagnosis. Eur J Intern Med. 2007 Mar;18(2):90-100. [CrossRef] [PubMed]

11. Diaz MH, Winchell JM. The evolution of advanced molecular diagnostics for the detection and characterization of Mycoplasma pneumoniae. Front Microbiol. 2016 Mar 8;7:232. [CrossRef] [PubMed]

12. Lee SC, Youn YS, Rhim JW, Kang JH, Lee KY. Early serologic diagnosis of Mycoplasma pneumoniae pneumonia: An observational study on changes in titers of specific-igm antibodies and cold agglutinins. Medicine. 2016 May;95(19):e3605. [CrossRef] [PubMed]

13. Lee WJ, Huang EY, Tsai CM, Kuo KC, Huang YC, Hsieh KS, et al. Role of serum Mycoplasma pneumoniae $\lg \mathrm{A}$, IgM, and $\lg \mathrm{G}$ in the diagnosis of mycoplasma pneumoniae-related pneumonia in school-age children and adolescents. Clin Vaccine Immunol. 2017 Jan 5;24(1). pii: e00471-16. [CrossRef] [PubMed]

14. Novacco M, Sugiarto S, Willi B, Baumann J, Spiri AM, Oestmann A, Riond B, et al. Consecutive antibiotic treatment with doxycycline and marbofloxacin clears 
bacteremia in Mycoplasma haemofelis-infected cats. Vet Microbiol. 2018 Apr;217:112-120. [CrossRef] [PubMed]

15. Valdivia L, Nix D, Wright M, Lindberg E, Fagan T, Lieberman D, Stoffer T, et al. Coccidioidomycosis as a common cause of community-acquired pneumonia. Send to Emerg Infect Dis. 2006 Jun;12(6):958-62. [CrossRef] [PubMed]

16. Wheat LJ. Approach to the diagnosis of the endemic mycoses. Clin Chest Med. 2009 Jun;30(2):379-89. [CrossRef] [PubMed]

17. Gupta N, Aragaki A, Wikenheiser-Brokamp KA, Benzaquen S, Panos RJ. Occam's razor or Hickam's dictum? J Bronchology Interv Pulmonol. 2012 Jul;19(3):216-9. [CrossRef] [PubMed] 